\title{
Management of a Rare Case of Adrenergic Myocarditis Complicated with Cardiogenic Shock
}

\author{
Liviu Macovei ${ }^{1,2}$, Robert Magopet ${ }^{1}$, Larisa Anghel ${ }^{1,2}$, \\ Mircea Ovanez Balasanian ${ }^{1,2}$, Cezara Raileanu ${ }^{1,2}$, \\ Dragos Marcu1,2, Cristina Prisacariu1,2, Cristian Statescu1,2
}

\section{Abstract}

A 41-year-old female was referred to our clinic with progressive dyspnea and a syncope, preceded by angina. On admission she was in cardiogenic shock. ECG showed diffuse repolarization changes and cardiac enzymes were elevated. The echocardiogram revealed severe left ventricular dysfunction with basal and medium walls hypokinesia. After stabilizing the patient, a coronary angiography was performed which revealed normal epicardial arteries. In the next days her clinical status was marked by severe hypertensive episodes with flash pulmonary edema and low responsiveness to therapy. Cardiovascular magnetic resonance showed myocardial edema and intramyocardial late gadolinium enhancement. An abdominal ultrasound raised suspicion of a pheochromocytoma due to an abnormal mass with cystic areas found on the right suprarenal gland. Elevated urinary free catecholamines and fractionated metanephrines confirmed the diagnosis. Further on, a CT scan better identified the heterogeneous tumor and the patient was referred for a right laparoscopic adrenalectomy. Followup at 1 month reported full recovery of the sistolic function. The particularity of the case is represented by the difficulty of diagnosis of adrenergic myocarditis, as well as the management of cardiogenic shock induced by it.

\section{Introduction}

Adrenergic myocarditis is a very rare presentation of pheochromocytoma and a particular cause of acute heart failure. Acute myocardial infarction, acute pulmonary edema, cardiac arrhythmias or
1 Institute of Cardiovascular Diseases "George I.M. Georgescu", lasi.

2 University of Medicine and Farmacy "Grigore T. Popa", lasi.

Contact information:

\section{Robert Magopet.}

Address: Institute of Cardiovascular Disease "George I.M. Georgescu", lasi, Romania. Boulevard Carol I, 50, lasi, 700503, Romania.

Tel: 0040232211834.

झ robert.magopet@gmail.com

Keywords

Adrenergic Myocarditis;

Pheochromocytoma;

Cardiogenic Shock. 
cadiomyopathies are only few of myocardial dysfunctions seen in this pathology. Myocarditis, as a clinically significant condition, is extremely rare in pheochromocytoma, and most likely is provoked by a direct myocardial injury caused by catecholamines [1]. Adrenergic myocarditis complicated with cardiogenic shock is a therapeutic challenge due to the important limitations of disease pathogenesis.

\section{Case report}

A 41-year-old female, former smoker (12 pack-years), was referred to our clinic with 2 days progressive dyspnea and a syncope preceded by chest pain. She had a personal history of hypertension for 1 year and she took nebivolol $2.5 \mathrm{mg}$ and lisinopril $10 \mathrm{mg}$, daily. On arrival, the patient was polypneic (24 breaths/minute), orthopneic, SpO2 $=88 \%$ with oxygen therapy and $\mathrm{FiO}_{2}$ of 0.45 , heart rate was 110 b.p.m. and blood pressure was $70 / 50 \mathrm{mmHg}$ on both arms. Cardiac auscultation revealed a systolic murmur in mitral area and pulmonary auscultation was specific with acute pulmonary edema - diffuse crackles in all lung fields. She had a BMI of $24 \mathrm{~kg} /$ $\mathrm{m}^{2}$ and was afebrile.

An ECG (Figure 1) was obtained showing sinus rhythm of 110 b.p.m.; intermediate heart axis and

Figure 1: ECG showed sinus rhythm, with diffuse repolarization changes.

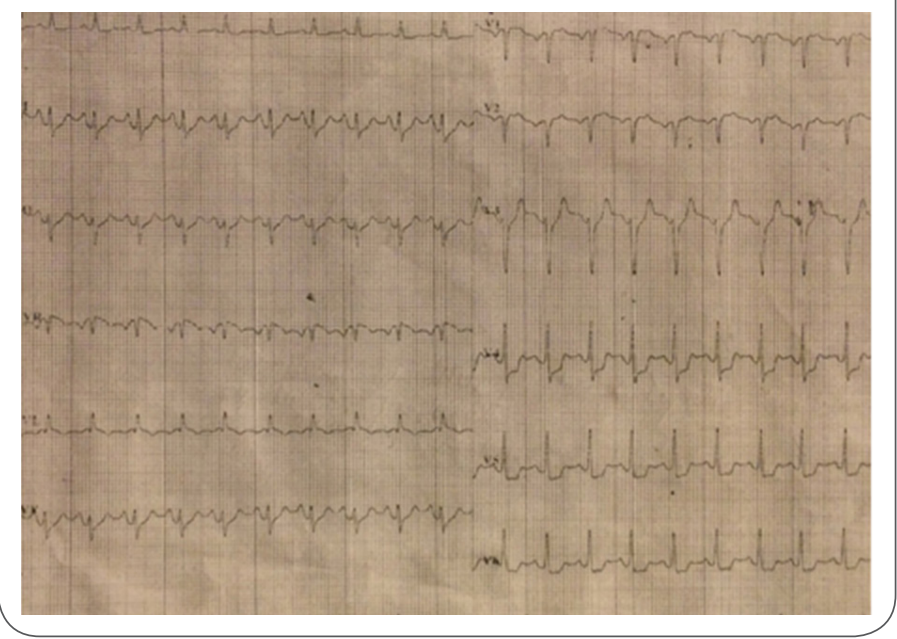

normal conduction times; $1 \mathrm{~mm}$ concave ST-segment elevations in lead aVR; $3 \mathrm{~mm}$ ST - segment depression in leads V4-V6, negative T wave in lead $\mathrm{aVL}$ and flat in D1.

The initial etiological differential diagnosis for the cardiogenic shock included acute coronary syndrome, myocarditis/Takotsubo syndrome, acute mitral regurgitation, aortic dissection.

Laboratory analyses exposed elevated cardiac enzymes (hs-cTnl =244ng/L, TGO 69 U/l, CK MB 76 $\mathrm{U} / \mathrm{I})$, normal lipid and renal profile, mild normochromic normocytic anemia $(\mathrm{Hg}=11.8 \mathrm{~g} / \mathrm{dl})$ but with normal iron levels. An echocardiogram was performed which revealed non dilated cardiac chambers: parasternal long axis view (PLAX): LV diastolic dimension $=45 \mathrm{~mm}$, LV systolic dimension=39mm; left atrium volume $=20 \mathrm{ml}$ (Simpson biplane method), parasternal short axis view (PSAX): RV diastolic dimension $=28 \mathrm{~mm}$, mild hypertrophy: PLAX: interventricular septum and posterior wall of $13 \mathrm{~mm}$ but with severely left ventricular systolic dysfunction (ejection fraction of $15 \%$ with Simpson biplane method), with akinetic basal walls and medium walls hypokinesia, moderate mitral regurgitation (PLAX: vena contracta $=5 \mathrm{~mm}$ ) with no pericardial effusion or aortic dissection.

We admitted the patient in the Intensive Cardiac Care Unit and began to treat accordingly the cardiogenic shock with acute pulmonary edema. We started with a continuous intravenous inotropic dobutamine support in dose of 5 micrograms/ $\mathrm{kg} / \mathrm{min}$ which was progressively increased to a dose of $20 / \mathrm{micrograms} / \mathrm{kg} / \mathrm{min}$ in 40 minutes in absence of not targeting a value of $65 \mathrm{mmHg}$ for the mean arterial pressure (MAP). At that time, we considered it necessary to initiate norepinephrine as a vasopressor adjunct to inotropic therapy in a dose of 2 micrograms/minute and we reached a MAP of $70 \mathrm{mmHg}$ with a dose of 5 micrograms/ minute (after 25 minutes). Nitroglycerine (5-15 micrograms/min) and furosemide (100 mg over 100 minutes) in continuous infusion protocol were also 
started along with morphine (single dose of $2 \mathrm{mg}$ ) and unfractionated heparin (initial i.v. bolus of 60 units/kg (4000 units), then i.v. infusion of 12 units/ $\mathrm{kg} / \mathrm{hr}$ ). Our main diagnosis at the arrival time was an acute coronary syndrome without ST- segment elevation and for this reason we also gave aspirin (250mg) and ticagrelor (180 mg) in loading doses with atorvastatin $80 \mathrm{mg}$. After a few hours, we observed a moderate improvement in the patient clinical status (remission of acute pulmonary edema: respiratory rate of $18 / \mathrm{min}, \mathrm{FiO}_{2}=0.3$, presence of diuresis, hemodynamic stability: MAP $=65-70$ $\mathrm{mmHg}$, normal pulmonary auscultation) so it was decided to continue with a coronary angiogram to exclude or treat significant obstructive coronary artery disease. Coronary angiography did not show obstructive coronary artery disease (Figure $2 \& 3$ ), and a left ventriculography (Figure 4) disclosed apical contractility but with mid-ventricular and basal akinesia, which raised suspicion of an inverted Takotsubo syndrome, although we did not have any information about a triggering stress event.

For the next 36 hours, the patient clinical status improved, with decreasing doses of dobutamine and norepinephrine until cessation.

Figure 2: Coronary angiography: left main coronary artery, left descending coronary artery and left circumflex coronary artery with no lesions.

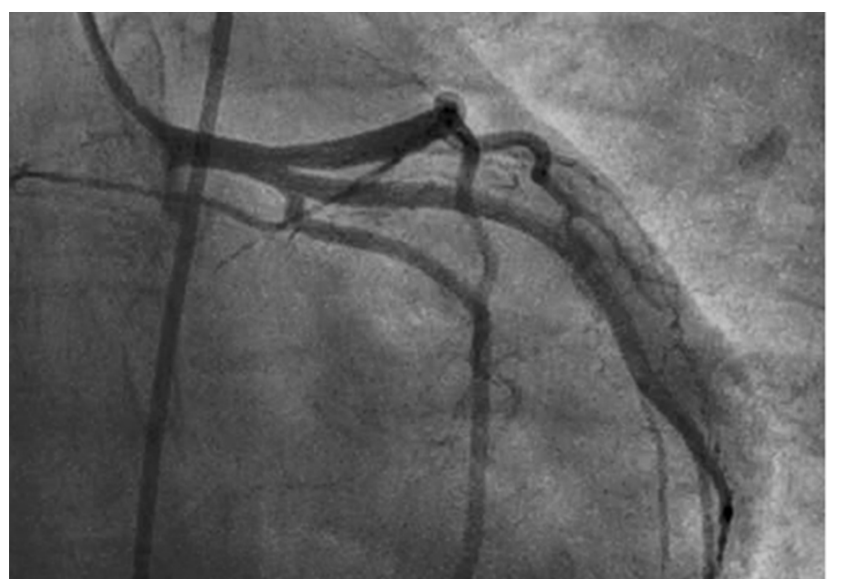

Figure 3: Coronary angiography. Right coronary artery with no lesions.

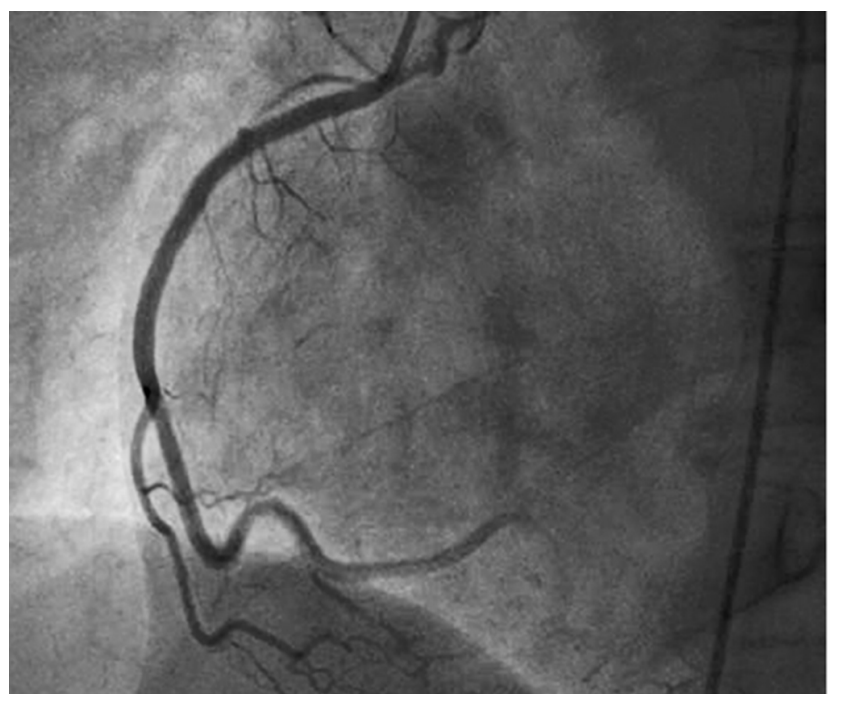

Figure 4: Left ventriculography. Apical contractility; mid-ventricular and basal akinesia.

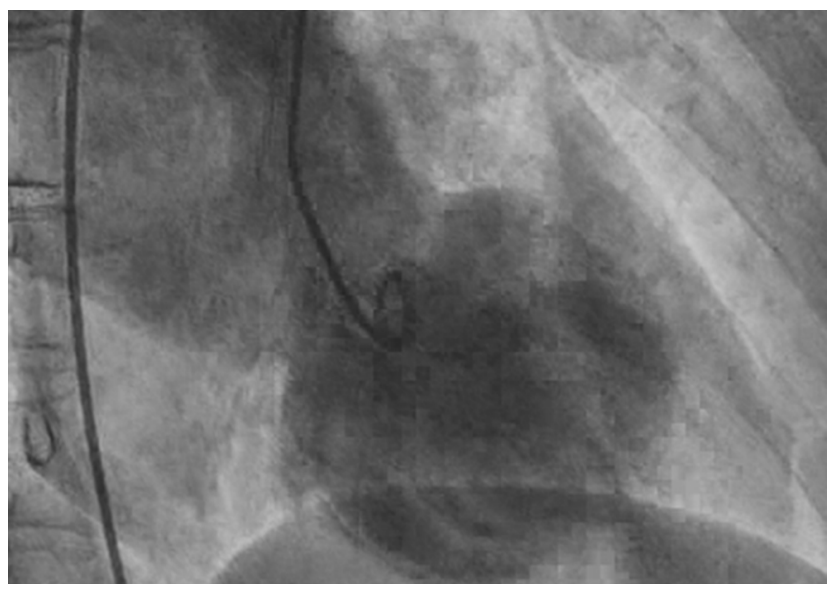

Due to these findings, the diagnosis was unclear and we referred the patient for a cardiovascular magnetic resonance study to better characterize the myocardial tissue. CMR revealed myocardial edema in the mid-ventricular and basal walls (T2-weighted imaging), while T1-weighted late gadolinium enhancement images showed diffuse myocardial enhancement, consistent with a non-ischemic myocardial damage. Taking into consideration the myocardial edema, increased wall thickness with severe hypokinesia and diffuse enhancement, a diagnosis of acute myocarditis was firmly established. 
After 76 hours from admittance the patient was again in acute pulmonary edema but with very high blood pressure $(250 / 130 \mathrm{mmHg})$ which required i.v. nitroglycerin in high doses (50 micrograms/minute), furosemide (200mg over 100 minutes), morphine (single dose of $2 \mathrm{mg}$ ) and urapidil (intravenous bolus at a dose of $12.5 \mathrm{mg}$, followed by a continuous infusion at a rate of $10 \mathrm{mg} / \mathrm{hr}$ ).

We performed a bedside abdominal ultrasound and noticed on the right adrenal gland topography a 50/34 mm tumor with cystic areas inside, highly suggestive of pheochromocytoma (Figure 5). Confirmation of diagnosis came when we dosed urinary free catecholamines and fractionated metanephrines on the second episode of flash pulmonary edema. (Urinary - metanephrine $1498 \mu \mathrm{g} / 24 \mathrm{~h}$ (N <300); normetanephrine $9720 \mu \mathrm{g} / 24 \mathrm{~h}$ ( $\mathrm{N}<400$ ); adrenaline $2133 \mu \mathrm{g} / 24 \mathrm{~h}(\mathrm{~N}<18)$; noradrenaline 314 $\mu \mathrm{g} / 24 \mathrm{~h}(\mathrm{~N}<83)$; dopamine $922 \mu \mathrm{g} / 24 \mathrm{~h} \mathrm{~h}(\mathrm{~N}<460)$.

Taking into consideration all of the above, the positive diagnosis of adrenergic myocarditis due to catecholamine excess was made. A CT abdominal scan better identified the heterogeneous tumor and the patient was referred for a right laparoscopic adrenalectomy. (Figure $6 \&$ 7). At that moment, the medical treatment included: alfa blocker (doxazosin $1 \mathrm{mg}$ o.d.), beta blocker (bisoprolol 5mg o.d.), dihydropyridine calcium channel blocker (amlodipine

Figure 5: Abdominal ultrasound revealed a solid, heterogeneous mass in the right suprarenal area.

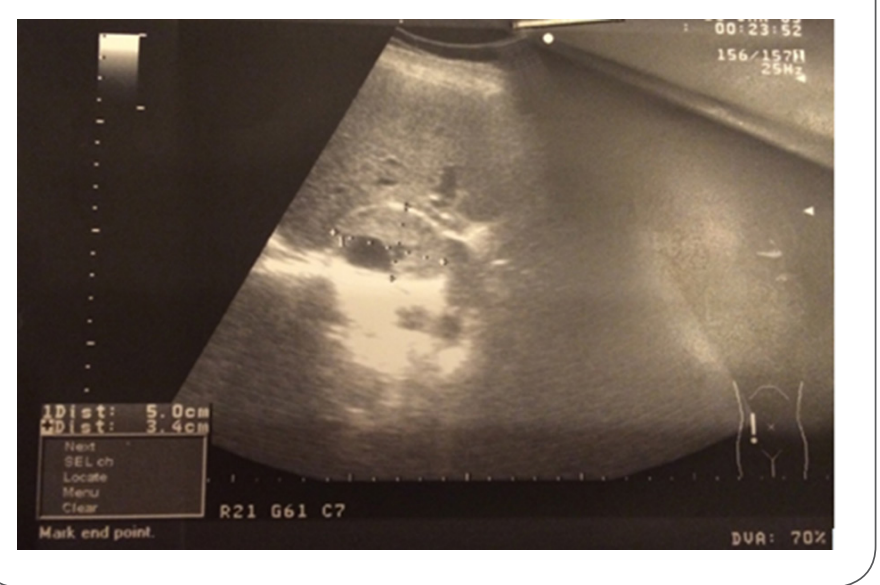

$10 \mathrm{mg}$ o.d.), angiotensin-converting-enzyme inhibitor (ramipril $10 \mathrm{mg}$ o.d.), diuretic (indapamide $1.5 \mathrm{mg}$ o.d.) and a mineralocorticoid receptor inhibitor (spironolactone $50 \mathrm{mg}$ o.d.) .

Follow-up at 1 month reported full recovery of the sistolic function (left ventricular ejection fraction of $55 \%$ ), with minimal antihypertensive therapy required (ramipril 2.5mg/o.d. and nebivolol 5mg/o.d).

Figure 6: Abdominal computed tomography disclosed an oval, well-defined right adrenal mass.

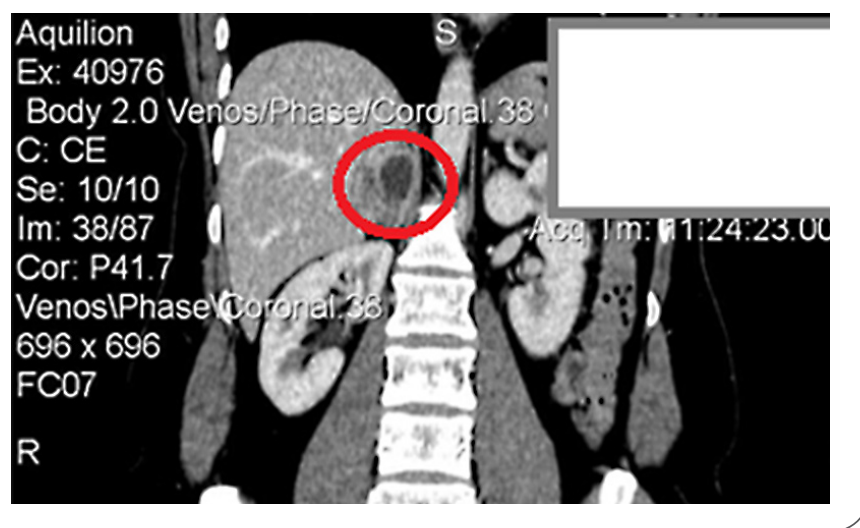

Figure7: Right adrenal gland with a massive tumor, surgically removed.

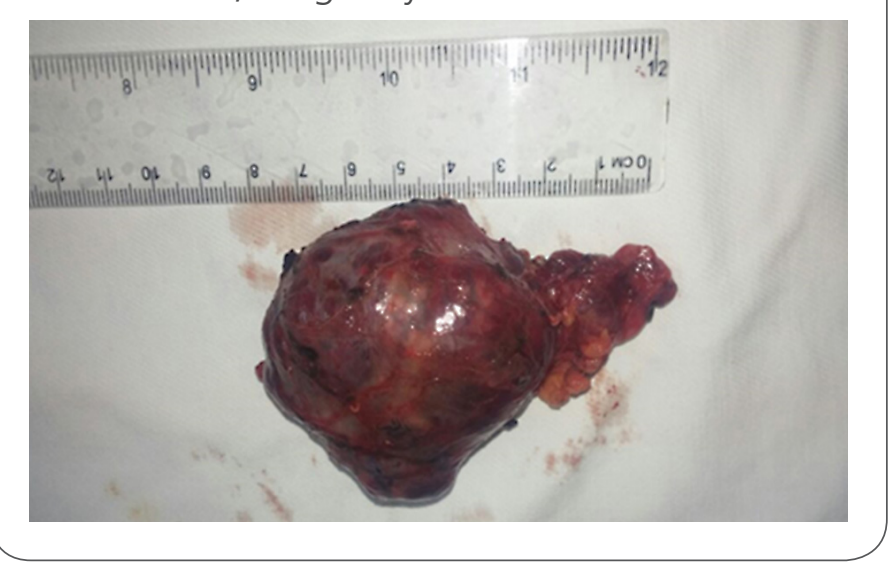

\section{Discussion}

We report a case of acute myocarditis in a young female, c omplicated with cardiogenic shock and flash pulmonary edema, in the context of an undiagnosed pheochromocytoma. Our case report provides direct evidence supporting the pathogenic role of 
excess catecholamine secretion in the development of adrenergic myocarditis.

Pheochromocytoma is a rare catecholamineproducing tumor, emerging from chromaffin cells of the adrenal medulla, with a vast majority of these tumors secreting both norepinephrine and epinephrine [2]. Palpitations, headaches and excessive sweating is the classic triad of this condition. Adrenergic myocarditis is a very rare presentation of pheochromocytoma and a particular cause of acute heart failure, with a pathophysiology which is not fully understood, although some autopsy studies revealed that myocardial inflammatory cell infiltrations and focal necrosis were found in 50\% of patients who died from pheochromocytoma $[3,4]$.

When facing a patient with cardiogenic shock of unknown origin, we must always take into consideration the presence of an underlying pheocromocytoma as a differential diagnosis, as well as other states of adrenergic hyperstimulation [5]. The reversibility of the myocardial affection in this phecromocytoma-associated myocardiopathy is common after the tumor resection [6].

At this moment, there is no effective standardized therapy for acute myocarditis, besides the optimal care of heart failure and arrhythmias in accordance with evidence-based guidelines and specific etiology-driven therapy $[7,8]$.

\section{Conclusion}

Adrenergic myocarditis, a rare pathological entity, represents a diagnostic and therapeutic challenge with important prognostic implications.

We believe that a multidisciplinary team (cardiologist, surgeon, radiologist, and anesthesiologist) is imperative in recognizing and managing these extremely difficult cases, while also discussing the therapeutic limitations induced by complications.

\section{References}

1. Sharkey $S$ et al. Takotsubo (Stress) Cardiomyopathy. Circulation. 2011; 124:e460-e462. https://www.ncbi.nlm.nih. gov/pubmed/22042929

2. Rostoff $P$ et al. Fulminant adrenergic myocarditis complicated by pulmonary edema, cardiogenic shock and cardiac arrest. Am J Emerg Med 2018 Feb; 36(2): 344.e1-344.e4. doi: 10.1016/j.ajem.2017.11.021. https://www.ncbi.nlm.nih.gov/ pubmed/29146416

3. Polito MV, Ravera A, Silverio A, Prota C, Lambiase C, Dellegrottaglie $S$, et al. A peculiar etiology of acute heart failure: adrenergic myocarditis. Am J Emerg Med 2015; 33(1545). https://www.ncbi.nlm.nih.gov/pubmed/26283614

4. Gravina M, Casavecchia G, D'Alonzo N, Totaro A,Manuppelli $V$, Cuculo A, et al. Pheochromocytoma mimicking Takotsubo cardiomyopathy and hypertrophic cardiomyopathy:a cardiac magnetic resonance study. Am J Emerg Med 2017; 35:353-355. https://www.ncbi.n/m.nih.gov/pubmed/27838037/

5. Esther Gil-Barrionuevo et. al. Adrenergic cardiomyopathy and cardiogenic shock as initial presentation of pheochromocytoma. A case report and review of the literature. International Journal of Surgery Case Reports. Volume 49, 2018,145-148. https:// www.ncbi.n/m.nih.gov/pmc/articles/PMC6068086/

6. R. Zhang, D. Gupta, S.G. Albert . Pheochromocytoma as a reversible cause of cardiomyopathy: analysis and review of the literature. Int. J. Cardiol., 15 December (249) (2017), pp. 319323. https://www.ncbi.n/m.nih.gov/pubmed/29121733

7. Skrzypiec-Spring $M$ et al. Carvedilol Inhibits Matrix Metalloproteinase-2 Activation in Experimental Autoimmune Myocarditis: Possibilities of Cardioprotective Application. J Cardiovasc Pharmacol Ther. 2018 Jan;23(1):89-97. doi: 10.1177/1074248417725058. https://www.ncbi.nlm.nih.gov/ pubmed/28854816

8. Bernahard Maisch. Cardio-Immunology of Myocarditis: Focus on Immune Mechanisms and Treatment Options. Front Cardiovasc Med. 2019; 6: 48. doi: 10.3389/fcvm. 2019.00048. https:// www.ncbi.nlm.nih.gov/pmc/articles/PMC6473396/

\section{Publish in International Archives of Medicine}

International Archives of Medicine is an open access journal publishing articles encompassing all aspects of medical science and clinical practice. IAM is considered a megajournal with independent sections on all areas of medicine. IAM is a really international journal with authors and board members from all around the world. The journal is widely indexed and classified Q2 in category Medicine. 